Article

\title{
Synchronization of Butterfly Fractional Order Chaotic System
}

\author{
Michal Fečkan ${ }^{1,2, *}$, T. Sathiyaraj ${ }^{3}(\mathbb{C})$ and JinRong Wang ${ }^{3,4}$ \\ 1 Department of Mathematical Analysis and Numerical Mathematics, Faculty of Mathematics, Physics and \\ Informatics, Comenius University in Bratislava, Mlynská dolina, 84248 Bratislava, Slovakia \\ 2 Mathematical Institute, Slovak Academy of Sciences, Štefánikova 49, 81473 Bratislava, Slovakia \\ 3 Department of Mathematics, Guizhou University, Guiyang 550025, Guizhou, China; \\ sathiyaraj133@gmail.com (T.S.); jrwang@gzu.edu.cn (J.W.) \\ 4 School of Mathematical Sciences, Qufu Normal University, Qufu 273165, Shandong, China \\ * Correspondence: Michal.Feckan@fmph.uniba.sk
}

Received: 22 February 2020; Accepted: 14 March 2020; Published: 19 March 2020

check for updates

\begin{abstract}
In this paper, we study the synchronization of a nonlinear fractional system, and analyze its time response and chaotic behaviors. We represent a solution for considered system by employing the Mittag-Leffler matrix function and Jacobian matrix. Thereafter, we prove synchronization of error system between drive-response systems using stability theory and linear feedback control methods. Finally, numerical simulations are presented to show the effectiveness of the theoretical results.
\end{abstract}

Keywords: fractional calculus; stability theory; synchronization

MSC: 34A08; 93C05

\section{Introduction}

Fractional calculus has over 300 years of history. Recently, it has attracted increasing interest due to its potential applications physics and engineering such as viscoelastic systems [1], dielectric polarization [2], electrode-electrolyte polarization [3], electromagnetic waves [4], quantitative finance [5], and quantum evolution of complex systems [6]. Synchronization of a chaotic system occurs when two or more chaotic systems are connected and it has attracted wide research because of its applications in science and engineering such as biology, economics, secure communication and signal generator design (see $[7,8]$ ). Sufficient conditions for synchronization of fractional-order chaotic systems via linear control were investigated in [9], synchronization of fractional chaotic systems with different orders was studied in [10] using stability concepts and synchronization of the Lotka-Volterra chaotic system was studied using the active control method in [11]. Robust synchronization for chaotic and hyperchaotic fractional order systems with model uncertainties and disturbances was investigated in [12].

Fractional chaotic system, global asymptotic synchronization and adaptive sliding mode synchronization have been studied in [13-15] and sufficient conditions were presented for exponential synchronization of fractional order chaotic systems in [16]. Finite time stability and synchronization of fractional order chaotic system with uncertainties and disturbance was studied in [17].

In this paper, we consider the following drive system of fractional order

$$
\left\{\begin{array}{l}
{ }^{C} D_{0}^{q} u(t)=A u(t)+f(u, u), \\
u(0)=u_{0}
\end{array}\right.
$$


and a corresponding response system of (1) is

$$
\left\{\begin{array}{l}
{ }^{C} D_{0}^{q} v(t)=A v(t)+f(u, v)+h(t), \\
v(0)=v_{0},
\end{array}\right.
$$

where $t \in \mathbb{R}_{+}:=[0, \infty), q \in(0,1),{ }^{C} D_{0}^{q}$ denotes the Caputo fractional derivative [18] with lower limit at $0, u(t) \in \mathbb{R}^{n}$ is a state, $A$ is a matrix of dimension $n \times n, f: \mathbb{R}^{n} \times \mathbb{R}^{n} \longrightarrow \mathbb{R}^{n}$ is a $C^{1}$-smooth and $h: \mathbb{R}_{+} \rightarrow \mathbb{R}^{n}$ is a controller.

Set the error system by $e(t)=v(t)-u(t)$. Define

$$
h(t)=B e(t)
$$

for an $n \times n$ matrix $B$. Then we get

$$
\left\{\begin{array}{l}
{ }^{C} D_{0}^{q} e(t)=(A+B+F(v, u)) e(t), \\
e(0)=e_{0}=v_{0}-u_{0}
\end{array}\right.
$$

where

$$
F(u, v)=\int_{0}^{1} \frac{\partial f}{\partial v}(u, \theta v+(1-\theta) u) d \theta
$$

is a Jacobian matrix.

From $[18,19]$ the solution $e(\cdot) \in C\left(\mathbb{R}_{+}, \mathbb{R}^{n}\right)$ of the system (4) is given by

$$
e(t)=E_{q}\left(t^{q} M\right) e_{0}+\int_{0}^{t}(t-s)^{q-1} E_{q, q}\left((t-s)^{q} M\right) F(v(s), u(s)) e(s) d s
$$

where $M=A+B, E_{q}(M)$ and $E_{q, q}(M)$ are the well-known Mittag-Leffler functions in [18,20,21].

We present sufficient conditions for synchronization of nonlinear fractional order chaotic system by using asymptotic stability theory for Mittag-Leffler matrix function, Jacobian matrix and linear feedback controller. We introduce a Jacobian matrix for the nonlinear term and represent the solution for error system between drive-response systems. Note that the time response of drive system (1) makes the impact in response system (2) (see the nonlinear term of (2)). Moreover, we numerically check the time response for drive-response systems for different fractional orders and employ the linear feedback controller to synchronize the drive-response systems based on the chaotic behavior and state trajectories.

\section{Main Results}

We give the following explicit results for the norm of Mittag-Leffler matrix functions.

Lemma 1. Assume

$$
M=\operatorname{diag}\left(-\lambda_{1},-\lambda_{2}, \cdots,-\lambda_{n}\right),
$$

for $0<\lambda_{1} \leq \lambda_{2} \leq \cdots \leq \lambda_{n}$. Then

$$
\left\|E_{q}\left(t^{q} M\right)\right\|=E_{q}\left(-t^{q} \lambda_{1}\right), \quad\left\|E_{q, q}\left((t-s)^{q} M\right)\right\|=E_{q, q}\left(-(t-s)^{q} \lambda_{1}\right) .
$$

Proof. From (7),

$$
E_{q}\left(t^{q} M\right)=\operatorname{diag}\left(E_{q}\left(-t^{q} \lambda_{1}\right), E_{q}\left(-t^{q} \lambda_{2}\right), \cdots, E_{q}\left(-t^{q} \lambda_{n}\right)\right)
$$

and

$$
E_{q, q}\left((t-s)^{q} M\right)=\operatorname{diag}\left(E_{q, q}\left(-(t-s)^{q} \lambda_{1}\right), E_{q, q}\left(-(t-s)^{q} \lambda_{2}\right), \cdots, E_{q, q}\left(-(t-s)^{q} \lambda_{n}\right)\right) .
$$


Since $E_{q}(-z)$ and $E_{q, q}(-z)$ are completely monotonous [21], we have

$$
\begin{aligned}
0<E_{q}\left(-t^{q} \lambda_{i}\right) & \leq E_{q}\left(-t^{q} \lambda_{1}\right), \\
0<E_{q, q}\left(-(t-s)^{q} \lambda_{i}\right) & \leq E_{q, q}\left(-(t-s)^{q} \lambda_{1}\right)
\end{aligned}
$$

for any $i=1,2, \cdots, n$ and $0 \leq s \leq t$. So considering any of the following standard norms on $\mathbb{R}^{n}$

$$
\|x\|=\sqrt{\sum_{i=1}^{n} x_{i}^{2}}, \quad\|x\|=\max _{i=1,2, \cdots, n}\left|x_{i}\right|, \quad\|x\|=\sum_{i=1}^{n}\left|x_{i}\right|,
$$

one can derive the results.

Definition 1. System (4) is called stable if for any $\epsilon>0$ there exists a $\delta>0$ such that $\left\|e_{0}\right\|<\delta$ guarantees that $\sup _{t \geq 0}\|e(t)\|<\epsilon$.

Definition 2. The drive system (1) is said to be synchronized with the response system (2), if there exists an error system (4) which is stable.

We introduce the following conditions:

[H1]: $\sup \|F(u, v)\|=l<\infty$. $(u, v) \in \mathbb{R}^{2 n}$

$[H 2]: M=A+B$ satisfies (7).

Theorem 1. Suppose that [H1] and [H2] hold. If $l<\lambda_{1}$ then the drive system (1) is synchronized with the response system (2) under the feedback controller (3).

Proof. From (6), [H1], [H2] and Lemma 1, we derive

$$
\|e(t)\| \leq E_{q}\left(-t^{q} \lambda_{1}\right)\left\|e_{0}\right\|+l \int_{0}^{t}(t-s)^{q-1} E_{q, q}\left(-(t-s)^{q} \lambda_{1}\right)\|e(s)\| d s .
$$

Set $\theta(\cdot)=\|e(\cdot)\|$. Introducing a linear bounded operator $\Theta: C\left(\mathbb{R}_{+}, \mathbb{R}_{+}\right) \rightarrow\left(\mathbb{R}_{+}, \mathbb{R}_{+}\right)$as

$$
(\Theta \theta)(t)=E_{q}\left(-t^{q} \lambda_{1}\right) \theta(0)+l \int_{0}^{t}(t-s)^{q-1} E_{q, q}\left(-(t-s)^{q} \lambda_{1}\right) \theta(s) d s .
$$

Then, (8) can be rewritten as

$$
\theta(t) \leq(\Theta \theta)(t), t \in \mathbb{R}_{+} .
$$

Since $\Theta$ is non-decreasing, we see that the sequence $\left\{\Theta^{k} \theta\right\}_{k=0}^{\infty}$ is non-decreasing having a limit $\theta_{\infty}$ which satisfies $\theta_{\infty}=\Theta \theta_{\infty}$, i.e.,

$$
\theta_{\infty}(t)=E_{q}\left(-t^{q} \lambda_{1}\right) \theta_{\infty}(0)+l \int_{0}^{t}(t-s)^{q-1} E_{q, q}\left(-(t-s)^{q} \lambda_{1}\right) \theta_{\infty}(s) d s,
$$

and $\theta(t) \leq \theta_{\infty}(t)$ for any $t \in \mathbb{R}_{+}$. But (9) means

$$
{ }^{C} D_{0}^{q} \theta_{\infty}(t)=-\lambda_{1} \theta_{\infty}(t)+l \theta_{\infty}(t)=\left(-\lambda_{1}+l\right) \theta_{\infty}(t),
$$

which has the solution

$$
\theta_{\infty}(t)=E_{q}\left(t^{q}\left(l-\lambda_{1}\right)\right) \theta_{\infty}(0)
$$

Summarizing, we obtain

$$
\|e(t)\| \leq E_{q}\left(t^{q}\left(l-\lambda_{1}\right)\right)\|e(0)\| .
$$


Further, from [22] (Lemma 2.5), (10) reduces to

$$
\|e(t)\| \leq \frac{m\left(q, 1, \lambda_{1}-l\right)}{t^{q}}\|e(0)\|
$$

where

$$
m\left(q, 1, \lambda_{1}-l\right)=\frac{\sin (\pi(1-q)) \int_{0}^{\infty} \exp \left(-r^{\frac{1}{q}}\right) d r}{\sin ^{2}(\pi q) \pi q\left(\lambda_{1}-l\right)}
$$

Finally, system (4) is stable with the convergence rate (11) and the drive system (1) is synchronized with the response system (2).

Remark 1. In [9-12,14-17], robust and exponential synchronization of fractional order deterministic chaotic systems was investigated using an adaptive scheme, an active control method, linear control, Lyapunov stability theory, linear feedback controller, sliding mode control and tracking control respectively. In this paper, we investigated the asymptotic synchronization for fractional order system using asymptotic stability theory, a feedback controller and Jacobian matrix.

Remark 2. (i) $[H 1]$ can be weakened in Theorem 1 to

$$
\left\|f\left(u, v_{1}\right)-f\left(u, v_{2}\right)\right\| \leq l\left\|v_{1}-v_{2}\right\|
$$

for any $u, v_{1}, v_{2} \in \mathbb{R}^{n}$.

(ii) The above synchronization is based on the stability of an equilibrium of a linear fractional equation ${ }^{c} D_{0}^{q} x(t)=M x(t)$. We know that this holds if and only if

$$
|\arg (\lambda)|>\frac{q \pi}{2}
$$

for any eigenvalue $\lambda$ of $M$. But then it is not so clear an estimate like (11). This is a reason, why we consider a diagonal M. For a general M satisfying (12), we must follow the way from Section 3.1 of [23], so a cumbersome approach based on real-valued Jordan form of $M$. Of course, the order in (7) is not important, so we can consider

$$
M=\operatorname{diag}\left(m_{11}, m_{22}, \cdots, m_{n n}\right)
$$

with $m_{i i}<0$ and take $\lambda_{1}=\max m_{i i}$.

Remark 3. Here, we proposed a key problem to study the synchronization of a nonlinear fractional order system by employing the Mittag-Leffler matrix function, Jacobian matrix, asymptotic stability theory and linear feedback control methods. The main advantages of the consider model is the time response of drive system (1) makes the effect in response system (2) and combined the nonlinear terms by using Jacobian matrix to represent a solution for error system. This type of model is more applicable and reasonable to study the stability concepts via stable equilibrium, point, state, manifold and finite and infinite dimensional stochastic settings. Further, as an applications point of view we show the behaviors of the considered model for different fractional order through numerical simulations.

\section{Examples}

In this section, we present the numerical examples to verify the obtained theoretical results.

Example 1. Consider the following fractional order drive system

$$
\left\{\begin{array}{l}
{ }^{C} D_{0}^{q} u(t)=A u(t)+f(u, u), t \geq 0, q \in(0,1), \\
u(0)=u_{0}
\end{array}\right.
$$


where

$$
A=\left(\begin{array}{ccc}
2 & 1 & 0 \\
0 & 4 & 1 \\
0 & 0 & -9
\end{array}\right)
$$

and

$$
u(t)=\left(u_{1}(t), u_{2}(t), u_{3}(t)\right)^{T}, \quad f(u, u)=\left(\begin{array}{c}
1 \\
\cos u_{2} \\
1
\end{array}\right)
$$

The state trajectory of the drive system (13) for different fractional orders $q=0.828,0.9,0.95,0.99$ are given in Figures 1-4. The trajectories of the system (13) is obviously stable for the fractional order $q<0.828$. From Figures 1-4, one can conclude that the behavior of the state trajectories $u_{1}(t), u_{2}(t), u_{3}(t)$ of the system (13) is unstable for the fractional order $q \geq 0.828$.
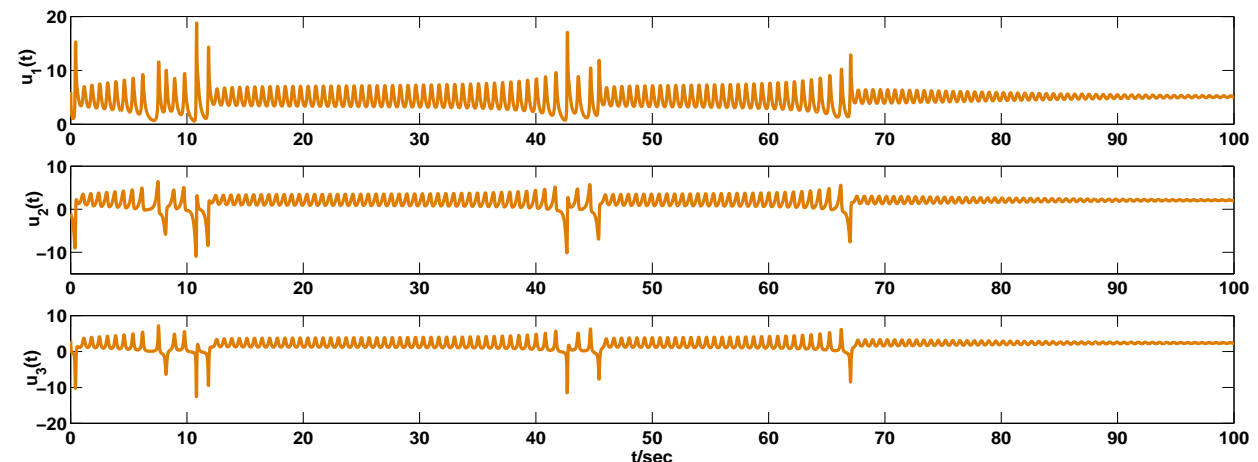

Figure 1. Time response of the states $u_{1}(t), u_{2}(t), u_{3}(t)$ of the drive system (13) with fractional order $q=0.828$.
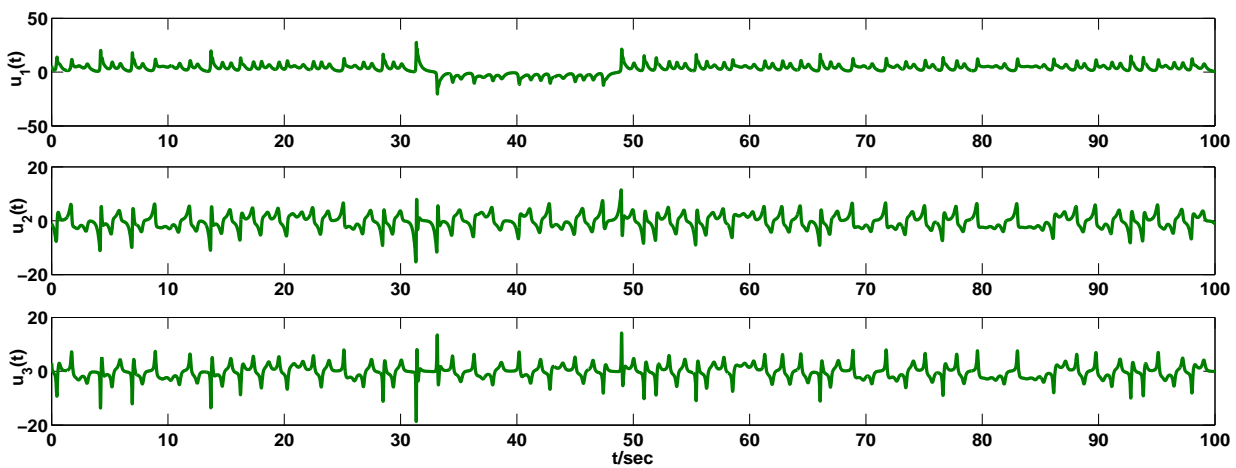

Figure 2. Time response of the states $u_{1}(t), u_{2}(t), u_{3}(t)$ of the drive system (13) with fractional order $q=0.9$. 


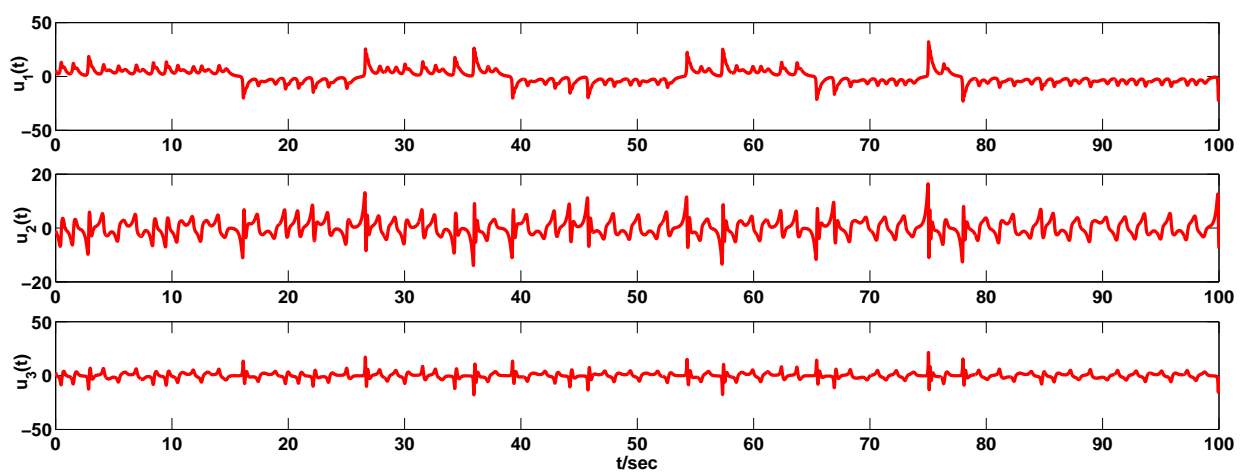

Figure 3. Time response of the states $u_{1}(t), u_{2}(t), u_{3}(t)$ of the drive system (13) with fractional order $q=0.95$.
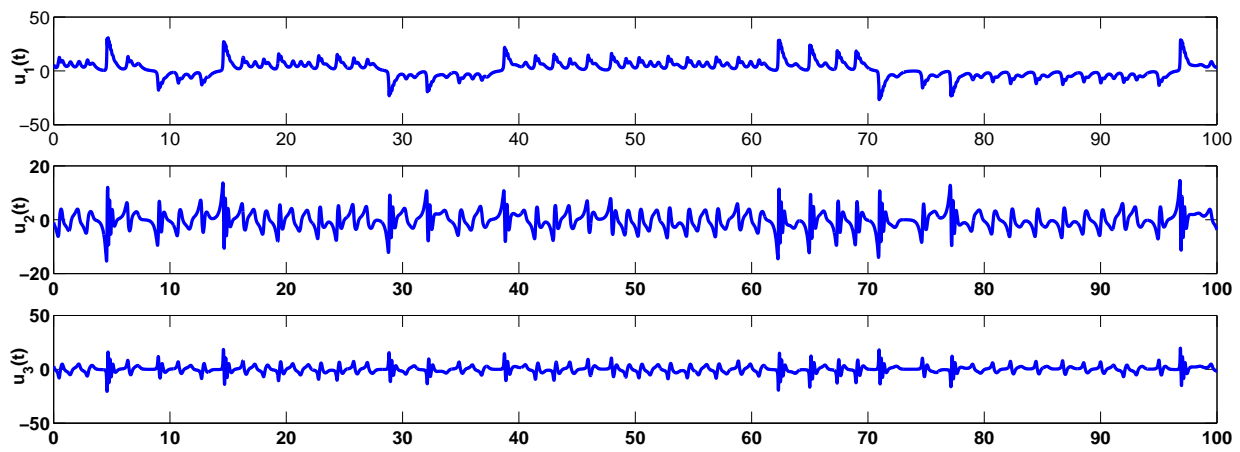

Figure 4. Time response of the states $u_{1}(t), u_{2}(t), u_{3}(t)$ of the drive system (13) with fractional order $q=0.99$.

Thus, it is necessary to introduce the control parameter $h(t)$ to synchronize the error system (15), which is given in the following Example 2.

Example 2. Consider the following fractional order response systems corresponding to the drive system (13)

$$
\left\{\begin{array}{l}
{ }^{C} D_{0}^{q} v(t)=A v(t)+f(u, v)+h(t), t \geq 0, q \in(0,1), \\
v(0)=v_{0},
\end{array}\right.
$$

and the errors between (13) and (14) satisfying

$$
\left\{\begin{array}{l}
{ }^{C} D_{0}^{q} e(t)=(A+B+F(v, u)) e(t), t \geq 0, q \in(0,1), \\
e(0)=e_{0}=v_{0}-u_{0}
\end{array}\right.
$$

where $M=A+B=\left(\begin{array}{ccc}-1 & 0 & 0 \\ 0 & -5 & 0 \\ 0 & 0 & -2\end{array}\right)$ and $\left(\lambda_{1}, \lambda_{2}, \lambda_{3}\right)=(-1,-5,-2)$,

$$
f(u, v)=\left(\begin{array}{c}
1+\frac{v-u}{2} \\
\cos u_{2} \\
1
\end{array}\right)
$$


and $A$ and $f(u, u)$ are already defined in Example 1. Further, as per Remark 2 (i), we can weakened in Theorem 1 to

$$
\left\|f\left(u, v_{1}\right)-f\left(u, v_{2}\right)\right\| \leq \frac{1}{2}\left\|v_{1}-v_{2}\right\| .
$$

Here, $l=\frac{1}{2}$ and satisfies the statement of the Theorem 1 i.e., $l<\lambda_{1}=1$.

Set the suitable control $h(t)=B e(t)$, step size $\hat{h}=0.001$ and $q=0.9$,

$$
h(t)=\left(h_{1}(t), h_{2}(t), h_{3}(t)\right)^{T}, B=\left(\begin{array}{ccc}
-3 & -1 & 0 \\
0 & -9 & -1 \\
0 & 0 & 7
\end{array}\right), e_{i}(t)=v_{i}(t)-u_{i}(t), i=1,2,3 .
$$

So

$$
\begin{aligned}
& h_{1}(t)=b_{11} e_{1}(t)+b_{12} e_{2}(t)+b_{13} e_{3}(t) ; b_{11}=-3, b_{12}=-1, b_{13}=0, \\
& h_{2}(t)=b_{21} e_{1}(t)+b_{22} e_{2}(t)+b_{23} e_{3}(t) ; b_{21}=0, b_{22}=-9, b_{23}=-1, \\
& h_{3}(t)=b_{31} e_{1}(t)+b_{32} e_{2}(t)+b_{33} e_{3}(t) ; b_{31}=0, b_{32}=0, b_{33}=7 .
\end{aligned}
$$

Next, from the matrices $f(u, u)$ and $f(u, v)$ we get $\|F(u, v)\|=l=\frac{1}{2}$. Hence, $[H 1]$ is verified by using the Jacobian matrix (5). The chaotic behavior of the states $\left(u_{1}(t), u_{2}(t)\right)$ and $\left(v_{1}(t), v_{2}(t)\right),\left(u_{1}(t), u_{3}(t)\right)$ and $\left(v_{1}(t), v_{3}(t)\right)$ and $\left(u_{2}(t), u_{3}(t)\right)$ and $\left(v_{2}(t), v_{3}(t)\right)$ of the drive-response systems (13) and (14) with fractional order $q=0.9$ are given in Figures $5-7$.

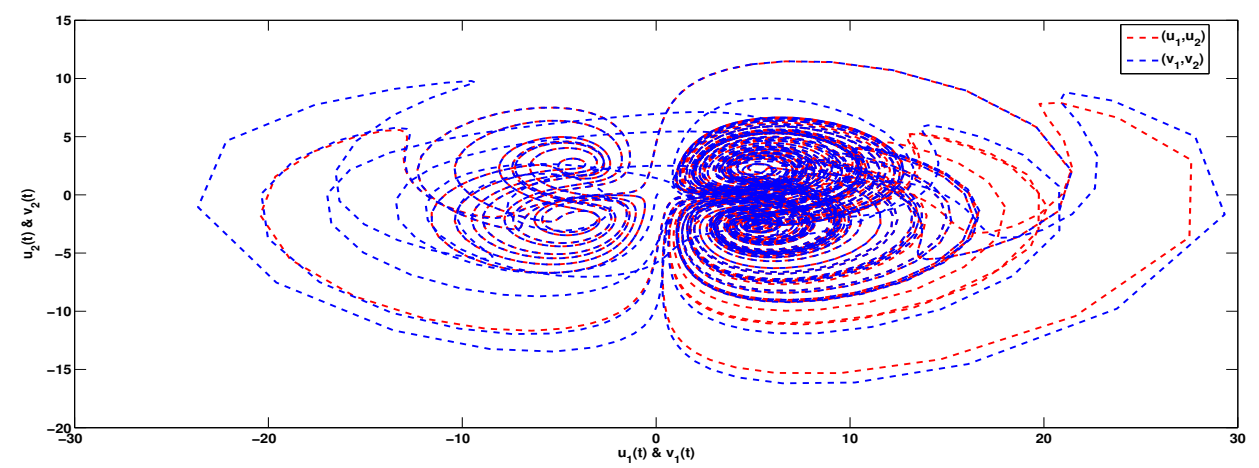

Figure 5. Chaotic behavior of the states $\left(u_{1}(t), u_{2}(t)\right)$ and $\left(v_{1}(t), v_{2}(t)\right)$ of the drive-response systems (13) and (14) with fractional order $q=0.9$, respectively.

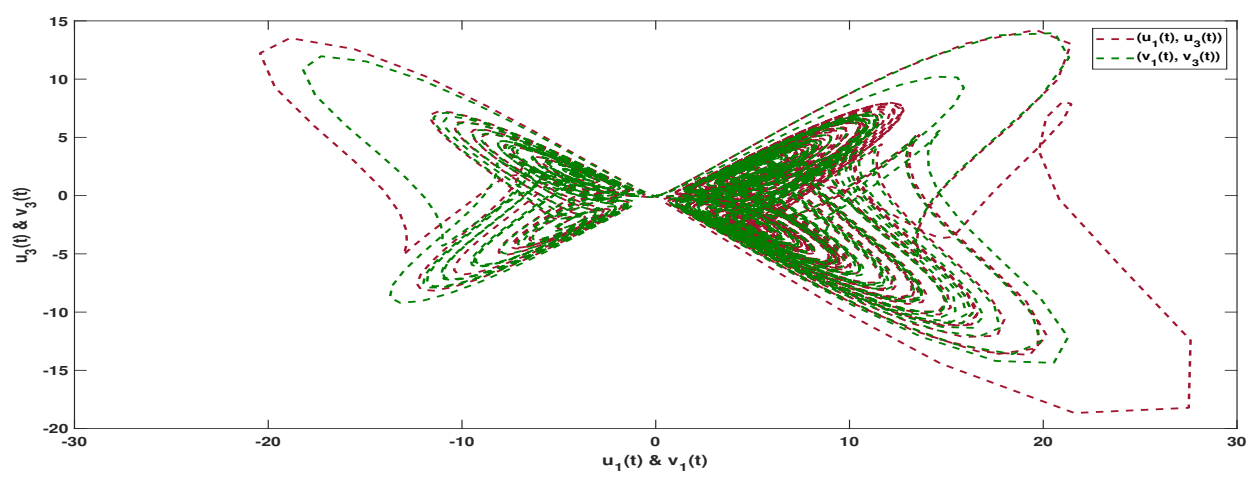

Figure 6. Chaotic behavior of the states $\left(u_{1}(t), u_{3}(t)\right)$ and $\left(v_{1}(t), v_{3}(t)\right)$ of the drive-response systems (13) and (14) with fractional order $q=0.9$, respectively. 


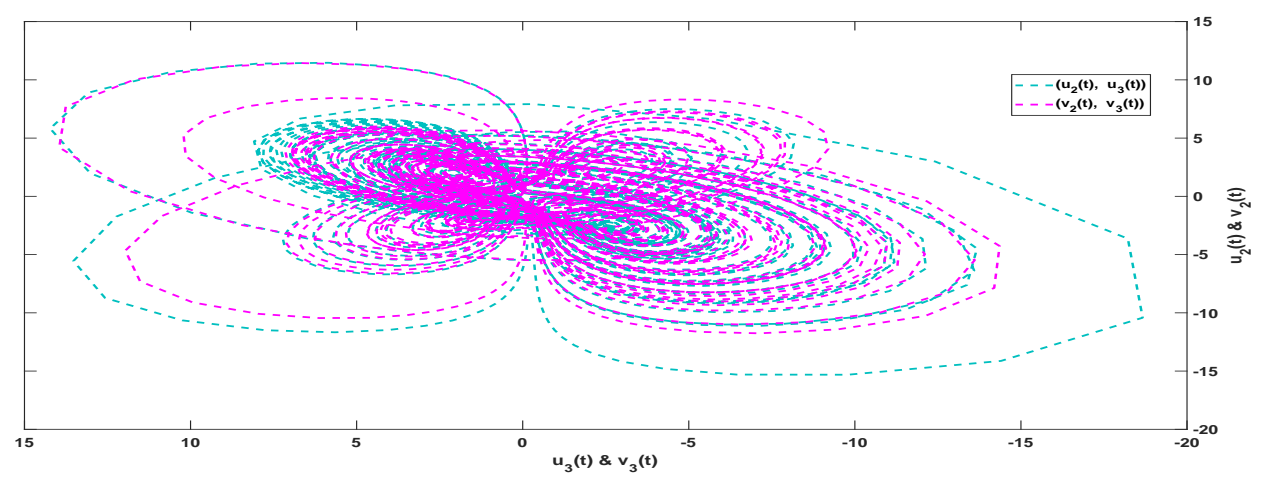

Figure 7. Chaotic behavior of the states $\left(u_{2}(t), u_{3}(t)\right)$ and $\left(v_{2}(t), v_{3}(t)\right)$ of the drive-response systems (13) and (14) with fractional order $q=0.9$, respectively.

Moreover, three-dimensional phase diagram of the states $u_{1}(t), u_{2}(t), u_{3}(t)$ of $(13)$ with fractional order $q=0.9$ and the time response of the states $\left(t, u_{1}(t)\right)$ and $\left(t, v_{1}(t)\right),\left(t, u_{2}(t)\right)$ and $\left(t, v_{2}(t)\right)$ and $\left(t, u_{3}(t)\right)$ and $\left(t, v_{3}(t)\right)$ of the drive-response systems (13) and (14) with fractional order $q=0.9$ are showed in Figures 8-11. In Figures 12-14, we have showed synchronized time response for the same states of the drive-response systems (13) and (14) with fractional order $q=0.9$. Finally, time response of the states $e_{1}(t), e_{2}(t), e_{3}(t)$ for the error system (15) with fractional order $q=0.9$ is showed in Figure 15.

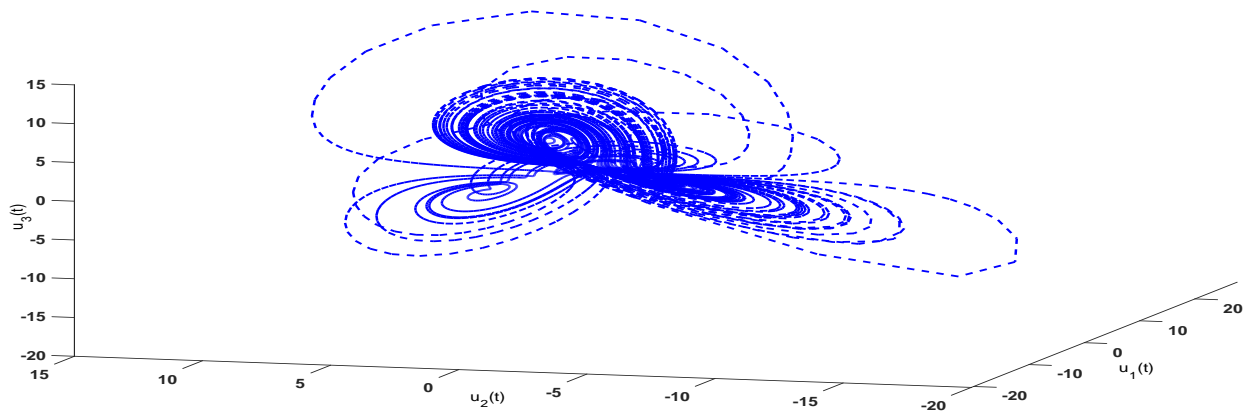

Figure 8. Three-dimensional phase diagram of the states $u_{1}(t), u_{2}(t), u_{3}(t)$ of the drive system (13) with fractional order $q=0.9$.

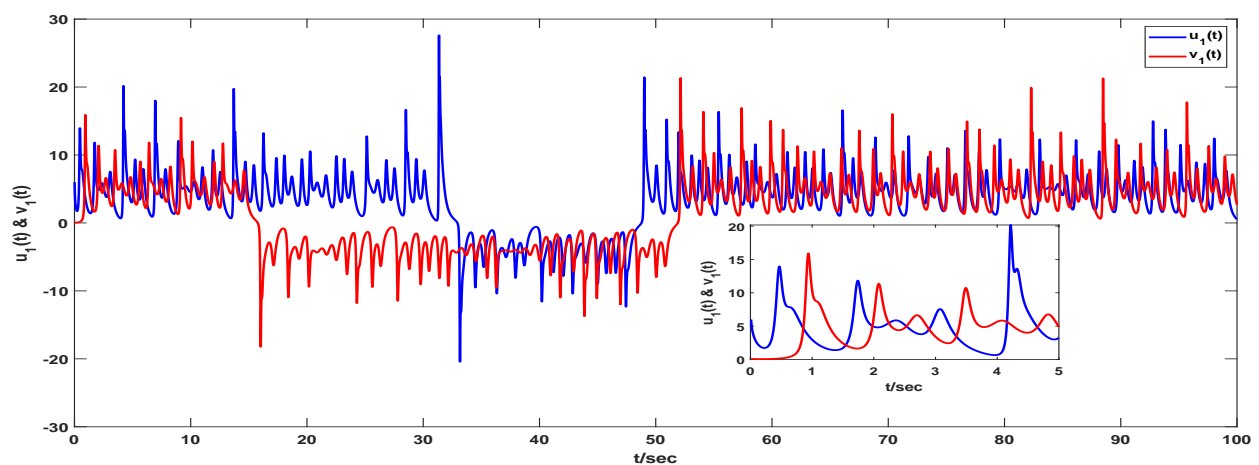

Figure 9. Time response of the states $\left(t, u_{1}(t)\right)$ and $\left(t, v_{1}(t)\right)$ of the drive-response systems (13) and (14) with fractional order $q=0.9$. 


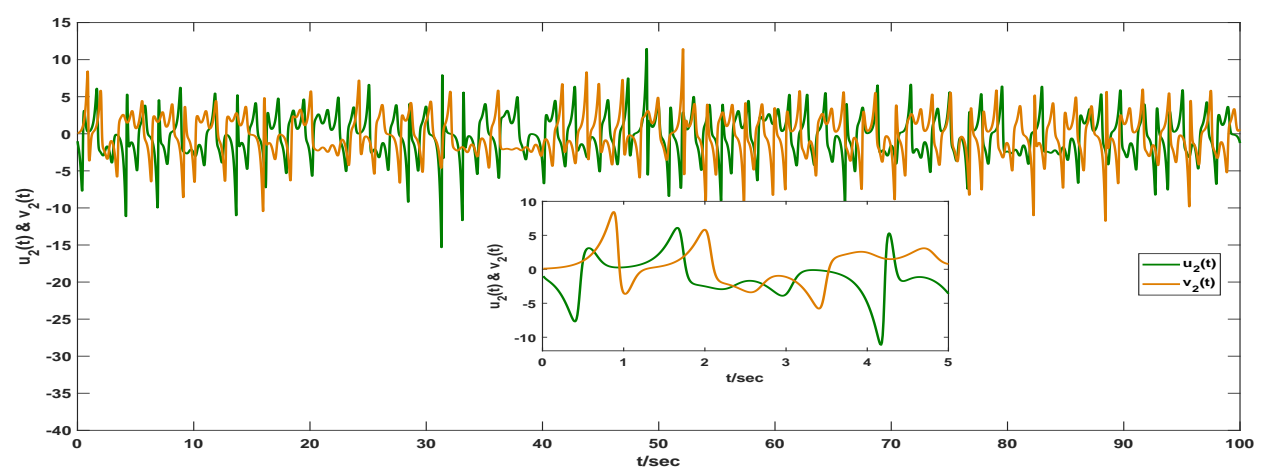

Figure 10. Time response of the states $\left(t, u_{2}(t)\right)$ and $\left(t, v_{2}(t)\right)$ of the drive-response systems (13) and (14) with fractional order $q=0.9$.

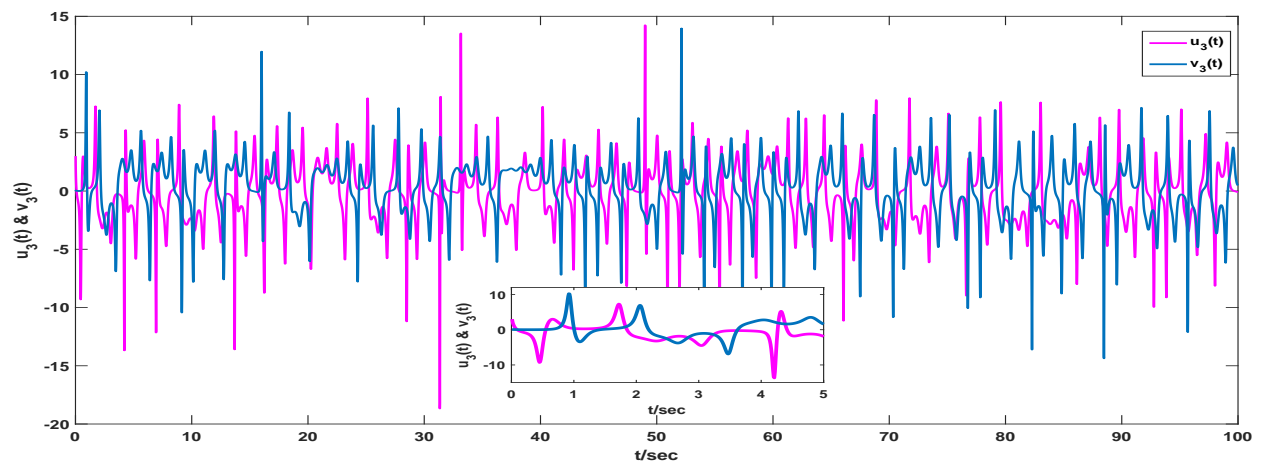

Figure 11. Time response of the states $\left(t, u_{3}(t)\right)$ and $\left(t, v_{3}(t)\right)$ of the drive-response systems (13) and (14) with fractional order $q=0.9$.

From Theorem 1, system (13) is synchronized with (14) under the control $h(t)$ as shown in the Figures 12-14. Time responses of the synchronization errors between (13) and (14) are shown in Figure 15, which provides the convergence of the synchronization errors to zero properly. Finally, all the hypothesis of Theorem 1 is verified numerically.

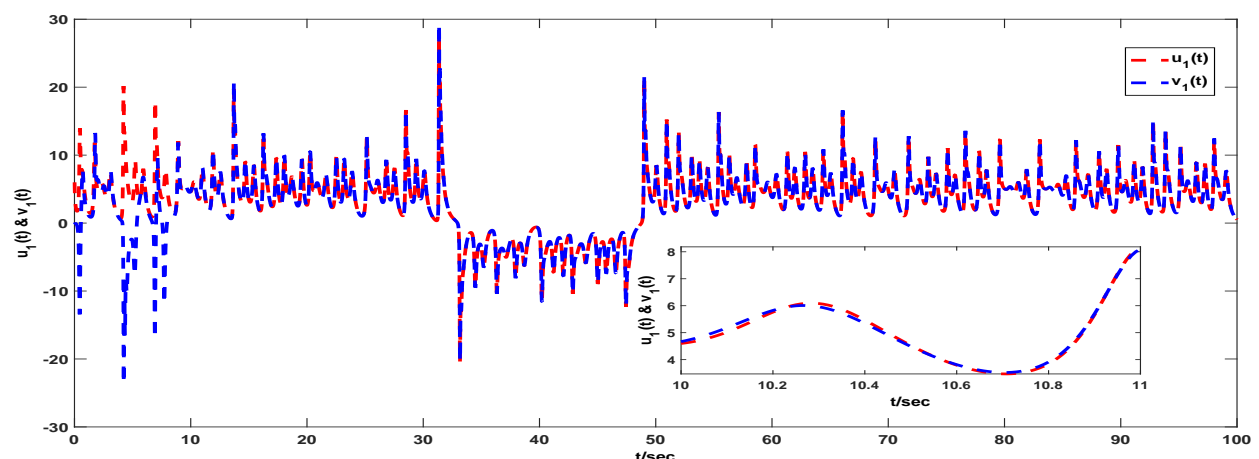

Figure 12. Synchronized time response of the states $\left(t, u_{1}(t)\right)$ and $\left(t, v_{1}(t)\right)$ of the drive-response systems (13) and (14) with fractional order $q=0.9$. 


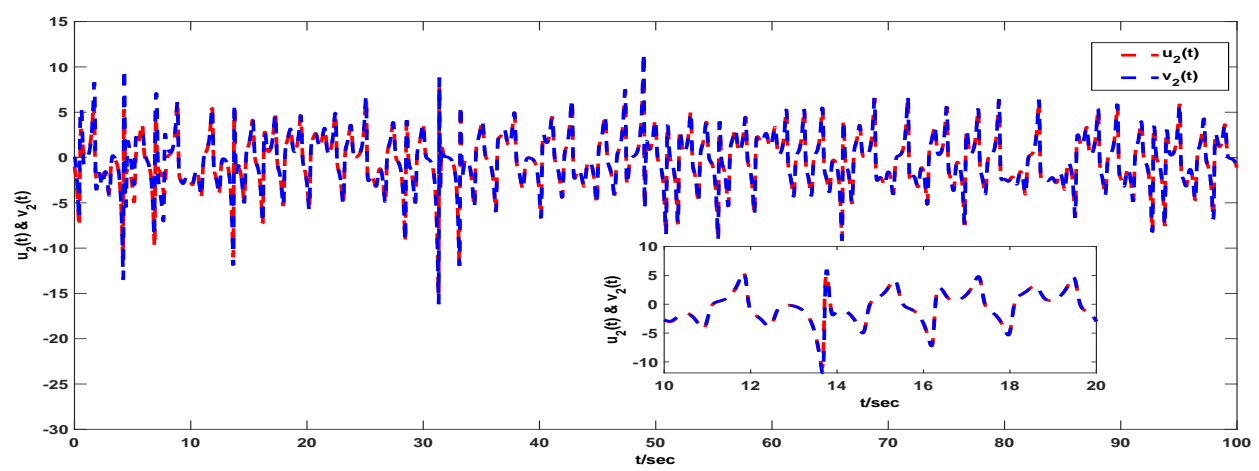

Figure 13. Synchronized time response of the states $\left(t, u_{2}(t)\right)$ and $\left(t, v_{2}(t)\right)$ of the drive-response systems (13) and (14) with fractional order $q=0.9$.

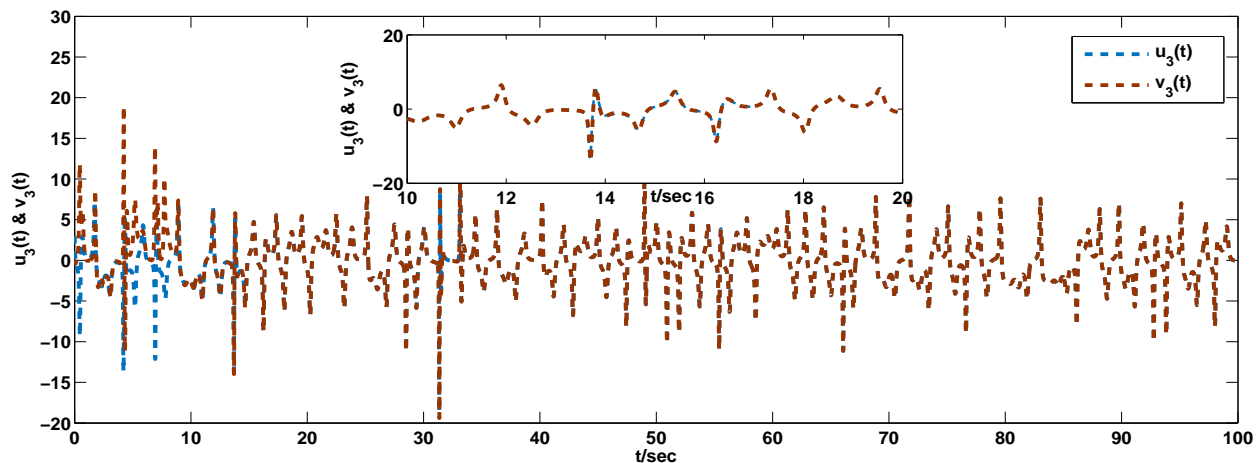

Figure 14. Synchronized time response of the states $\left(t, u_{3}(t)\right)$ and $\left(t, v_{3}(t)\right)$ of the drive-response systems (13) and (14) with fractional order $q=0.9$.

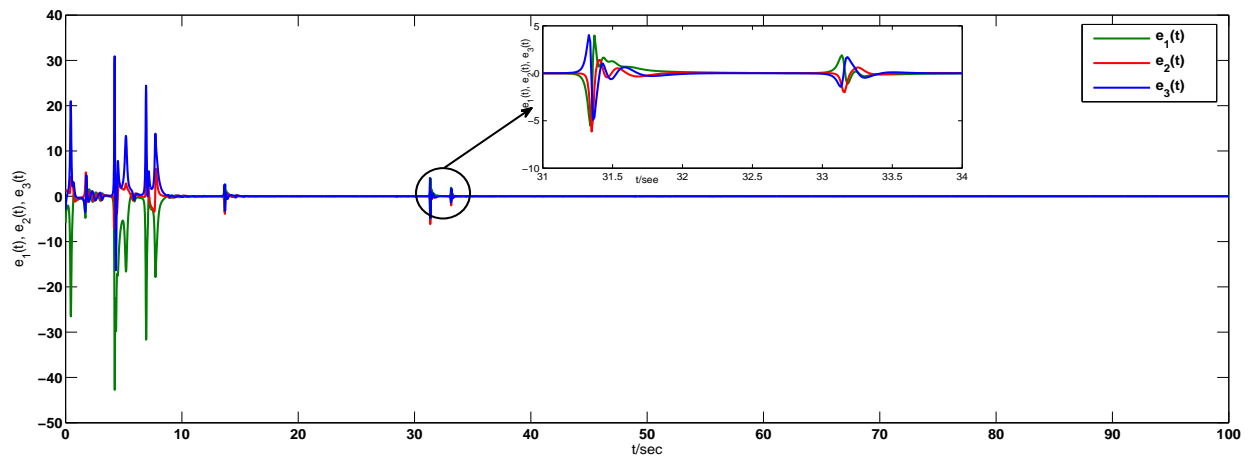

Figure 15. Time response of the states $e_{1}(t), e_{2}(t), e_{3}(t)$ for the error system (15) with fractional order $q=0.9$.

\section{Conclusions}

We presented synchronization criteria for nonlinear fractional order systems using Jacobian matrix and asymptotic stability estimation of the Mittag-Leffler matrix function, and a suitable linear feedback controller. The above arguments can be extended to fractional evolution equations of (1) and (2) with (3) on a Banach space $X$ when $A: D(A) \subseteq X \rightarrow X$ is a generator of a $C_{0}$-semigroup $\left\{S_{A}(t), t \geq 0\right\}$ on $X$ [24], $f: X \times X \rightarrow X$ is globally Lipschitz, $h: \mathbb{R}_{+} \rightarrow X$ is continuous and $B: X \rightarrow X$ is linear and continuous. We intend to study this case in our next paper. 
Author Contributions: M.F. and J.W. contributed to the supervision and project administration, M.F., T.S. and J.W. contributed to the conceptualization and methodology. All authors have read and approved the final manuscript.

Funding: This work is partially supported by the National Natural Science Foundation of China (11661016), Training Object of High Level and Innovative Talents of Guizhou Province ((2016)4006), Major Research Project of Innovative Group in Guizhou Education Department ([2018]012), Guizhou Data Driven Modeling Learning and Optimization Innovation Team ([2020]5016), the Slovak Research and Development Agency under the contract No. APVV-18-0308, and the Slovak Grant Agency VEGA No. 1/0358/20 and No. 2/0127/20.

Acknowledgments: The authors thanks the referees for their careful reading of the article and insightful comments.

Conflicts of Interest: The authors declare no conflict of interest.

\section{References}

1. Bagley, R.L.; Calico, R.A. Fractional order state equations for the control of viscoelastically damped structures. J. Guid. Control. Dyn. 1991, 14, 304-311. [CrossRef]

2. Sun, H.H.; Abdelwahab, A.A.; Onaral, B. Linear approximation of transfer function with a pole of fractional power. IEEE Trans. Autom. Control 1984, 29, 441-444. [CrossRef]

3. Ichise, M.; Nagayanagi, Y.; Kojima, T. An analog simulation of non-integer order transfer functions for analysis of electrode processes. J. Electroanal. Chem. Interfacial Electrochem. 1971, 33, 253-265. [CrossRef]

4. Heaviside, O. Electromagnetic Theory; Chelsea: New York, NY, USA, 1971.

5. Laskin, N. Fractional market dynamics. Physica A 2000, 287, 482-492. [CrossRef]

6. Kusnezov, D.; Bulgac, A.; Dang, G.D. Quantum Lévy Processes and Fractional Kinetics. Phys. Rev. Lett. 1999, 82, 1136-1139. [CrossRef]

7. Milanovic, V.; Zaghloul, M.E. Synchronization of chaotic neural networks and applications to communications. Int. J. Bifurc. Chaos 1996, 6, 2571-2585. [CrossRef]

8. Pecora, L.M.; Carroll, T.L. Synchronization in chaotic systems. Phys. Rev. Lett. 1990, 64, 821-825. [CrossRef] [PubMed]

9. Odibat, Z.M.; Corson, N.; Aziz-Alaoui, M.A.; Bertelle, C. Synchronization of chaotic fractional-order systems via linear control. Int. J. Bifurc. Chaos 2010, 20, 81-97. [CrossRef]

10. Zhou, P.; Ding, R. Chaotic synchronization between different fractional-order chaotic systems. J. Frankl. Inst. 2011, 348, 2839-2848. [CrossRef]

11. Agrawal, S.K.; Srivastava, M.; Das, S. Synchronization of fractional order chaotic systems using active control method. Chaos Solitons Fractals 2012, 45, 737-752. [CrossRef]

12. Li, C.; Su, K.; Tong, Y.; Li, H. Robust synchronization for a class of fractional-order chaotic and hyperchaotic systems. Optik 2013, 124, 3242-3245. [CrossRef]

13. Hu, T.; Zhang, X.; Zhong, S. Global asymptotic synchronization of nonidentical fractional-order neural networks. Neurocomputing 2018, 313, 39-46. [CrossRef]

14. Shao, S.; Chen, M.; Yan, X. Adaptive sliding mode synchronization for a class of fractional-order chaotic systems with disturbance. Nonlinear Dyn. 2016, 83, 1855-1866. [CrossRef]

15. Wang, Q.; Qi, D.L. Synchronization for fractional order chaotic systems with uncertain parameters. Int. J. Control. Autom. Syst. 2016, 14, 211-216. [CrossRef]

16. Mathiyalagan, K.; Park, J.H.; Sakthivel, R. Exponential synchronization for fractional-order chaotic systems with mixed uncertainties. Complexity 2015, 21, 114-125. [CrossRef]

17. Li, C.; Zhang, J. Synchronization of a fractional-order chaotic system using finite-time input-to-state stability. Int. J. Syst. Sci. 2016, 47, 2440-2448. [CrossRef]

18. Kilbas, A.A.; Srivastava, H.M.; Trujillo, J.J. Theory and Applications of Fractional Differential Equations; Elsevier: Amsterdam, The Netherlands, 2006.

19. Li, K.; Peng, J. Laplace transform and fractional differential equations. Appl. Math. Lett. 2011, 24, $2019-2023$.

20. Podlubny, I. Fractional Differential Equations, Mathematics in Science and Engineering; Academic Press: San Diego, CA, USA, 1999; Volume 198.

21. Goreno, R.; Kilbas, A.A.; Mainardi, F.; Rogosin, S.V. Mittag-Leffler Functions, Related Topics and Applications; Springer: Berlin, Germany, 2014.

22. Peng, S.; Wang, J. Existence and Ulam-Hyers stability of ODEs involving two Caputo fractional derivatives. Electron. J. Qual. Theory Differ. Equ. 2015, 52, 1-16. [CrossRef] 
23. Cong, N.D.; Doan, T.S.; Siegmund, S.; Tuan, H.T. On stable manifolds for fractional differential equations in high-dimensional spaces. Nonlinear Dyn. 2016, 86, 1885-1894. [CrossRef]

24. Pazy, A. Semigroup of Linear Operators and Applications to Partial Differential Equations; Springer: New York, NY, USA, 1983.

(C) 2019 by the authors. Licensee MDPI, Basel, Switzerland. This article is an open access article distributed under the terms and conditions of the Creative Commons Attribution (CC BY) license (http://creativecommons.org/licenses/by/4.0/). 